\title{
Avaliação de sistemas de produção de batata orgânica na região Sul do Rio Grande do Sul
}

\author{
Lírio J Reichert ${ }^{1}$; Mário C Gomes²; José Ernani Schwengber ${ }^{1}$; Arione da S Pereira ${ }^{1}$ \\ 'Embrapa Clima Temperado, C. Postal 403, 96010-971 Pelotas-RS; lirio.jose@embrapa.br; jose.ernani@embrapa.br; arione.pereira@ \\ embrapa.br; ${ }^{2} \mathrm{UFPel} /$ Depto. Ciências Sociais, Pelotas-RS; mconill@gmail.com
}

\begin{abstract}
RESUMO
O cultivo de batata no município de São Lourenço do Sul-RS, ocorre desde a chegada dos primeiros imigrantes alemães e pomeranos, há mais de 150 anos. Seu cultivo sempre teve importância socioeconômica expressiva, devido suas tradições, hábitos e cultura trazida pelos colonizadores, promovendo o desenvolvimento não só deste município, mas de toda a região Sul do RS. Entretanto, a partir da década de 1990 a bataticultura dessa região passou a enfrentar vários problemas tecnológicos e estruturais, perdendo espaço para outros cultivos. Uma alternativa encontrada por um grupo de agricultores foi desenvolvê-la em sistema de produção orgânico. Neste sentido, o objetivo deste trabalho foi avaliar sistemas de produção de batata orgânica, visando identificar os critérios que os agricultores julgam importantes neste sistema de produção. Foram selecionados dois grupos de agricultores que cultivam batata no sistema orgânico. Um grupo formado por agricultores que fazem parte do projeto de pesquisa Rede de Referência da Embrapa Clima Temperado, e outro grupo formado por agricultores de fora da Rede. Utilizou-se a metodologia multicritério de apoio à decisão (MCDA), para a construção dos modelos de uma forma participativa e interativa. De maneira geral, os modelos construídos não se diferenciaram muito em relação às práticas de produção adotadas em cada um dos grupos de agricultores. No entanto, diferenças foram observadas nos níveis de impacto das ações e no estabelecimento de pesos diferentes entre os modelos, de modo que o resultado final, na ordem de preferência dos critérios de avaliação, foi diferente. Para o grupo da rede de referência, tamanho da batata foi o critério mais importante, enquanto para o grupo fora da rede foi o critério riscos de produção.
\end{abstract}

Palavras-chave: Solanum tuberosum, agricultura familiar, produção ecológica, metodologia multicritério.

\begin{abstract}
Evaluation of organic potato production systems in the southern Rio Grande do Sul State, Brazil

The production of potatoes in the municipality of São Lourenço do Sul, in the state of Rio Grande do Sul, Brazil, occurs since the arrival of the first German and Pomeranian immigrants for over 150 years. This crop has always had significant socioeconomic importance, for its tradition, habit and culture brought by the colonizers, not only promoting the development of this municipality, but also the entire southern RS. However, from the 1990s on, the potato crop of this region faced several technological and structural problems, losing ground to other crops. An alternative found by a group of farmers is the organic production system. In this sense, the objective of this work was to evaluate the potato organic systems in order to identify criteria that the farmers consider important in this production system. Two groups of farmers that grow potatoes in the organic system were selected. A group of farmers of the Reference Network of Embrapa Clima Temperado research project, and another group formed by farmers outside the Reference Network. The multicriteria decision support methodology (MCDA) was used to create participatory and interactive models. In general, the models constructed did not differ much in relation to the production practices adopted by each group of farmers. However, differences were observed in the levels of impact of actions and in the establishment of different weights between models, so the end result in order of preference of the evaluation criteria was different. For the group belonging to the reference network, potato size was the most important criterion, while for the group outside the network was the criterion risk of production.
\end{abstract}

Keywords: Solanum tuberosum, family farm, ecological production, multicriteria methodology.

(Recebido para publicação em 25 de julho de 2012; aceito em 10 de abril de 2013) (Received on July 25, 2012; accepted on April 10, 2013)

$\mathrm{P}$ ara os consumidores, a batata (Solanum tuberosum) pode ser vista como mais um alimento levado à mesa, porém para os agricultores ela é uma fonte de renda para mantê-los no campo. Para a pesquisa, ela representa uma fonte de investigação seja no campo do melhoramento genético ou na melhoria de uma prática de cultivo. Para o comércio, ela representa uma fonte de renda com transações diárias numa relação direta com o consumidor. No setor produtivo, envolvem a organização de agricultores por meio de associações, cooperativas, órgãos de pesquisa e assistência técnica em prol do desenvolvimento de sistemas de produção tecnicamente viáveis. Enfim, são vários os segmentos que se beneficiam dela e por estes e outros motivos, tornou-se um dos alimentos mais consumidos por suas qualidades nutricionais.

O Rio Grande do Sul (RS) já foi o maior produtor de batatas do Brasil e hoje ocupa o $4^{\circ}$ lugar, estando à sua frente Minas Gerais, São Paulo e Paraná (FNP CONSULTORIA \& AGROINFORMATIVOS, 2011). No RS, a produção atualmente está distribuída em várias regiões; no entanto, no passado, ela concentrava-se no Sul do Estado, basicamente na região compreendida pelos municípios de Pelotas, Canguçu, Morro Redondo, São Lourenço do Sul e Cristal.

São Lourenço do Sul teve, num 
passado recente, na produção de batata uma das atividades agrícolas de maior expressão econômica. Trazida pelos imigrantes alemães e pomeranos, esta atividade talvez tenha sido a que mais projetou o município além de suas fronteiras. Conforme descreve Coaracy (1957), a batata era vendida para os mercados de Pelotas, Rio Grande, Rio de Janeiro, Montevideo e Buenos Aires, onde encontrava boa aceitação. Costa (1984) relata que nas décadas de 1940 e 1950, São Lourenço do Sul tinha o título de maior produtor de batatas da América Latina, o que contribuiu para que o Rio Grande do Sul se tornasse o maior produtor do Brasil.

A produção continuou crescendo no município até o ano de 1992, quando atingiu seu ponto mais elevado em área cultivada, com 12.000 ha e a produção de 91.000 t, em duas safras anuais. Após aquele ano teve início um processo de queda do cultivo motivado por vários fatores tecnológicos, ambientais, econômicos, mercadológicos e pela desorganização dos produtores, de modo que em 2009, a produção foi tão somente de 15.900 t, com uma área cultivada de apenas 1.300 ha. Muitos agricultores mudaram a matriz produtiva para outros cultivos entre eles o tabaco, como relataram Pereira \& Daniels (2003), Madail et al. (2005), Lima (2006) e Martinez (2009).

Segundo Pereira \& Daniels (2003), a redução de área em São Lourenço do Sul foi ocasionada por fatores tais como os custos crescentes de produção, a baixa capacidade competitiva em relação às produções do centro do país, e o elevado padrão do produto no mercado nacional, obtido pelo uso de elevada tecnologia incompatível com o modelo da agricultura familiar. Madail et al. (2005) apontam ainda a defasagem do conhecimento dos agricultores sobre o mercado da batata e a lenta adoção de tecnologias de produção para atender às demandas do mercado consumidor. Lima (2006) comenta que os elementos da paisagem como o solo e o clima, além dos fatores históricos culturais, foram no passado os que potencializaram o desenvolvimento da bataticultura no município e que hoje, esses mesmos elementos da paisagem são inibidores dessa produção. Martinez
(2009) salienta que os produtores não souberam tratar questões do mercado e sua evolução, ficando refém dos atravessadores.

Enfim, estes e outros motivos, fizeram com que muitos agricultores abandonassem o cultivo da batata, de modo que atualmente não mais representa a principal fonte de renda agrícola na área colonial de São Lourenço do Sul.

Visando mudar esse quadro e, pela importância da batata não somente como alimento essencial, mas também pelos aspectos econômicos, sociais e culturais que esta hortaliça representa para os agricultores deste município, várias iniciativas surgiram no sentido de alavancar o seu cultivo, resgatando as tradições e conhecimentos transmitidos de geração a geração.

Para mudar o quadro descrito, um conjunto de entidades como a Embrapa Clima Temperado, a Emater-RS, o Centro de Apoio ao Pequeno Agricultor (CAPA) e cooperativas (Cooperativa Mista dos Pequenos Agricultores da região Sul - COOPAR e Sul Ecológica de Agricultores Familiares) estão unindo esforços com o objetivo de retomar o plantio da batata em São Lourenço do Sul. O processo tem evoluído por meio do desenvolvimento de tecnologias de produção orgânica e de aplicação dos conceitos e fundamentos da Agroecologia. De acordo com Leff (2002), nesses sistemas há um intercâmbio de experiências livre e espontânea que aproxima o agricultor do técnico, potencializando os conhecimentos de quem a pratica. Altieri (1998) por sua vez, afirma que a Agroecologia fornece uma estrutura metodológica de trabalho para a compreensão mais profunda tanto da natureza como dos princípios de funcionamento do agroecossistema. Segundo o autor, a Agroecologia integra os princípios agronômicos, ecológicos e socioeconômicos numa visão multidimensional; incentiva os pesquisadores a penetrarem no conhecimento e nas técnicas dos agricultores e juntos desenvolverem sistemas mais sustentáveis dos pontos de vista econômico, social e ambiental.

Visualizando o mercado de produtos orgânicos ou agroecológicos, um grupo de agricultores do município, apoiados pelas suas organizações e pelo trabalho da pesquisa, estão mudando seu sistema de cultivo convencional para o orgânico. Neste sentido, a pesquisa tem desenvolvido novas cultivares mais adaptadas a este sistema, tem modificado práticas culturais, como o manejo de pragas e doenças, da fertilização da área e, principalmente, na oferta de sementes de boa qualidade. Algumas dessas tecnologias são encontradas em Nazareno \& Pereira (2009) e Pereira \& Daniels (2003).

Recentemente, a Embrapa disponibilizou novas cultivares de batata, visando oferecer ao produtor materiais que atendam às exigências do consumidor, que preza por um produto de qualidade e de boa aparência. Aliado aos aspectos de qualidade que interessam aos consumidores, as novas cultivares também apresentam boa resistência à requeima (Phytophtora infestans) que, segundo Arce (2002), é a doença fúngica que mais causa danos na batata no mundo inteiro. Nazareno \& Jaccoud Filho (2003) afirmam que, na região Sul do Brasil, esta também tem sido a maior preocupação dos agricultores. Rossi (2009) relata que no sistema de produção orgânico, a pinta-preta juntamente com a requeima são as doenças que mais causam danos à lavoura, visto que elas reduzem a área foliar e o ciclo de desenvolvimento vegetativo, comprometendo a produtividade e a qualidade da batata.

Com vistas a atender o mercado de batata orgânica, oferecendo um produto mais saudável para os consumidores e às suas famílias, um grupo de agricultores familiares da região Sul do RS, vem aperfeiçoando os sistemas de produção de batata no sistema orgânico. Com o objetivo de avaliar sistemas de produção de batata orgânica desenvolvidos por bataticultores, o estudo identificou os critérios utilizados pelos mesmos e a sua importância, analisando seus pontos de estrangulamento, suas organizações, seus canais de comercialização, que têm na batata uma fonte geradora de renda promovendo o desenvolvimento e o crescimento socioeconômico da unidade familiar.

\section{MATERIAL E MÉTODOS}

O trabalho foi desenvolvido no mu- 
nicípio de São Lourenço do Sul, com quatro agricultores familiares que cultivam a batata no sistema orgânico e/ou de base ecológica no segundo semestre do ano de 2010.

A região de estudo está enquadrada na divisão territorial do MDA/SDT como Território Zona Sul, que é formado por 25 municípios, localizado na região Sul do $\mathrm{RS}$, sendo caracterizada por um forte vínculo da produção agropecuária oriunda da categoria da agricultura familiar. $\mathrm{O}$ território possui uma área geográfica de $38.066,5 \mathrm{~km}^{2} \mathrm{e}$ uma população de 864.343 habitantes, dos quais 151.738 vivem no meio rural, o que representa $18 \%$ da população (IBGE, 2010). A região está limitada a leste pelo Oceano Atlântico, a oeste pela Região da Campanha, ao norte faz divisa com a margem direita do Rio Camaquã (Amaral Ferrador e Cristal) e ao Sul faz fronteira com o território da Republica Oriental do Uruguai. Geograficamente está situado entre os meridianos de longitude $50^{\circ} 45^{\prime}$ e $54^{\circ} 30^{\prime} \mathrm{O}$ e entre os paralelos $30^{\circ} 30^{\prime}$ e $33^{\circ} 50^{\prime} \mathrm{S}$.

Para a avaliação, foi utilizada a ferramenta de Apoio à Decisão (MCDA - Multi Criteria Decision-Aid), conforme descrita por Ensslin et al. (2001). Esta metodologia aplica, segundo Roy (1993), uma abordagem construtivista num processo participativo e interativo entre os decisores e os facilitadores envolvidos. O pressuposto básico do MCDA é reconhecer a importância da subjetividade dos decisores, ou seja, que não é possível excluir do processo de apoio à decisão aspectos, tais como, valores, objetivos, preconceitos, cultura e intuição (Roy \& Vanderpooten, 1996). Trata-se de uma ferramenta de apoio à tomada de decisão que auxilia na resolução de problemas complexos e assume uma relação de ajuda entre um facilitador e um decisor, e permite apoiar o processo em busca da decisão satisfatória, que, neste caso específico, serão os agricultores em um processo de avaliação do sistema de produção de batata orgânica.

É uma metodologia de exploração de ideias, visando à obtenção das melhores soluções de um grupo de pessoas para auxiliar a tomada de decisão na resolução de um problema. Xavier (2010) avaliou sistemas de produção de milho com agricultores de assentamentos da reforma Agrária na região de Unaí-MG. Anderson (2010) identificou quais critérios os agricultores utilizam no momento da compra de um trator para sua unidade de produção. São apenas dois exemplos de trabalhos realizados com grupos de agricultores e que auxiliaram no processo de tomada de decisão.

Para a realização deste estudo, foram selecionados dois grupos de agricultores que desenvolvem seus cultivos de batata no sistema orgânico. Um grupo formado por agricultores que fazem parte do projeto de pesquisa "Rede de Referência da Embrapa Clima Temperado" (GRR) Resultados dessas pesquisas podem ser encontradas em Medeiros et al. (2005). O outro grupo foi formado por agricultores associados à Cooperativa Sul Ecológica de Agricultores Familiares, à Associação Regional de Produtores Agroecologistas da Região Sul (ARPA-Sul) de Pelotas e a COOPAR, de São Lourenço do Sul, identificados como grupo fora da rede de referência (GFRR). Foram aplicados 34 questionários, sendo 14 no GRR e 20 no GFRR, com o objetivo de caracterizá-las, e selecionar quatro, sendo duas em cada grupo, pelo critério da renda bruta $(\mathrm{RB})$ obtida na batata perante a RB total da unidade. As quatro unidades selecionadas estão localizadas em São Lourenço do Sul.

Os passos e etapas para a busca dos dados, definição das unidades e construção dos modelos foram realizados em quatro etapas: aplicação dos questionários no período de setembro 2009 a janeiro 2010; processamento e análise dos dados para selecionar as quatro unidades com o auxílio do software Excel; reuniões de coleta de dados para a construção dos modelos de avaliação da produção de batata orgânica, realizadas no período de outubro 2010 a janeiro 2011; e reuniões de avaliação e validação dos modelos com os respectivos grupos de agricultores, realizadas em março 2011.

Durante o processo de construção dos modelos, foram resgatadas as etapas do processo de produção de batata orgâ- nica. As reuniões foram gravadas com o prévio consentimento dos agricultores, visando manter uma memória do processo, evitando perda de informações importantes na construção dos modelos. De acordo com Ensslin et al. (2001), o processo de construção do modelo é dividido em três fases: estruturação do problema, avaliação das ações potenciais e elaboração de recomendações.

Fase de estruturação do modelo - A construção do modelo iniciou pela identificação do contexto decisório, que foram as quatro unidades agrícolas. Nesta fase foi organizado o conhecimento dos diversos atores sobre as dimensões de avaliação relevantes, chamadas de Pontos de Vista Fundamentais (PVF) ou “critérios". Segundo Keeney (1992), em uma estrutura arborescente. Os atores, que são pessoas que têm interesse direto ou indireto na decisão (Ensslin et al., 2001), são classificados em dois tipos: os "decisores", que são aqueles que participam diretamente do processo decisório, e os "facilitadores", que têm o papel de apoiar os "decisores" de forma não neutra na construção do modelo multicritério.

Para sistematizar a representação dos agricultores sobre a situação, foi empregada a técnica do mapeamento cognitivo (Eden, 1989). O mapa cognitivo é uma representação gráfica de uma representação mental, que o pesquisador (facilitador) organiza a partir de uma imaginação discursiva, formulada pelo sujeito (ator) sobre um objeto (o problema). Sua construção obedece a uma hierarquia de conceitos, relacionados por ligações de influencia entre meios e fins (Montibeller Neto, 1996).

Para a sua construção, foi definido um rótulo para o problema, que na concepção adotada é definido como uma situação que se deseja alterar, mas não há muita segurança de como obter essa alteração. Para a identificação do problema, os agricultores foram questionados sobre os aspectos que são considerados importantes para avaliar sistemas de produção de batata orgânica. As respostas foram obtidas por meio de uma sessão de brainstorm $^{1}$, que compuseram

\footnotetext{
${ }^{1}$ É uma metodologia de exploração de ideias, visando a obtenção das melhores soluções de um grupo de pessoas para a resolução de um problema.
} 
o conjunto de Elementos Primários de Avaliação (EPAs).

A transição do mapa cognitivo para a estrutura do modelo na forma arborescente foi feita por meio da identificação dos pontos de vista fundamentais (PVFs) para avaliação, empregando-se a técnica do enquadramento de Keeney (1992). O uso dessa técnica consistiu na identificação de clusters de análise no mapa cognitivo e dentro dos clusters ramos e linhas de argumentação entre os conceitos.

Fase de avaliação - Nessa fase, os PVFs foram operacionalizados por meio da construção de descritores ou atributos, segundo Keeney (1992), para que, sobre eles, fosse possível identificar o impacto de qualquer ação a ser considerada. Um descritor é definido como um conjunto de níveis de impacto, organizados em uma escala de ordem decrescente de preferência, de tal forma que o impacto medido seja estabelecido de forma não ambígua (Ensslin et al., 2001). São definidos os níveis mínimos e máximos de cada descritor. O nível mínimo representa a situação menos desejável, mas possível de ocorrer e o nível máximo, ao contrário, representa a situação mais desejada, mas não idealizada e, portanto, de difícil ocorrência. Posteriormente, são estabelecidos os níveis bom e neutro de cada descritor, isto é, aqueles que delimitam a região de competitividade das alternativas a serem avaliadas.

As funções de valor locais associadas a cada nível de impacto são construídas utilizando-se o método Direct Rating (Beinat, 1995). Segundo o mesmo autor, para a mensuração de preferências são utilizadas escalas de intervalos. A principal característica deste tipo de escala é ter o zero e a unidade de medida arbitrária. Por isso, as únicas transformações admissíveis que preservam as propriedades representadas da escala original são as transformações lineares positivas do seguinte tipo:

$$
\begin{aligned}
& \mathrm{v}^{\prime}(a)=\mathrm{a} \cdot \mathrm{v}(a)+\mathrm{b}, a \mathrm{~A} \\
& \text { Onde: } \\
& \mathrm{v}^{\prime}(a)=\text { novo valor para a ação } a \\
& \mathrm{v}(a)=\text { antigo valor para a ação } a \\
& \mathrm{a}, \mathrm{b} \quad \text { e a }>0 \\
& \mathrm{~A}=\text { conjunto de ações potenciais }
\end{aligned}
$$

Uma vez que dois pontos da nova escala estão sendo fixados (o zero e o 100), a definição da nova escala será obtida pela resolução de um pequeno sistema de equações.

$$
\begin{aligned}
& 100=\mathrm{a} \cdot \mathrm{v}(\text { bom })+\mathrm{b} \\
& 0=\mathrm{a} \cdot \mathrm{v}(\text { neutro })+\mathrm{b}
\end{aligned}
$$

Onde: $\mathrm{v}($ bom $)=$ valor do impacto do nível bom na escala antiga; v(neutro) = valor do impacto do nível neutro na escala antiga

Resolvendo-se para a e b, obtém-se os parâmetros necessários para transformar a escala inteira.

Após esse procedimento, cada PVF é reconhecido como um critério de avaliação, que é definido como uma função matemática que mede o desempenho de ações potenciais de forma menos ambígua possível, a performance das ações de acordo com um eixo ou ponto de vista considerado por um decisor ou grupo de decisores (Bouyssou, 1990).

Esses critérios, por sua vez, foram agregados em uma função de valor global aditiva, utilizando-se taxas de harmonização ou weights (Keeney, 1992), que foram obtidas a partir das preferências comparativas dos decisores por cada PVF, empregando-se o método Swing Weights (Gomes, 2001). Normalizar os níveis neutro e bom é necessário também para que as taxas de harmonização possam ser consideradas como fatores de escala nessa função de agregação. Este procedimento pode ser utilizado para representar a quantificação dos trade-off envolvidos nas diversas estratégias em avaliação. Operacionalmente, sugeriuse uma situação hipotética que todos os descritores tivessem impacto em todos os critérios no nível neutro. Solicitou-se, então, que os agricultores pensassem em uma alteração nesta situação, na qual eles escolheriam um critério a ser alterado para o nível bom em primeiro lugar. A essa alteração foi estabelecida uma pontuação igual a 100, que corresponde à taxa de harmonização bruta do critério.

Foi feito o mesmo procedimento considerando o critério a ser elevado do nível neutro para o bom em segundo lugar, e apresentando-se a seguinte questão para os agricultores: Se o critério escolhido em primeiro lugar recebeu uma pontuação igual a 100, qual deveria ser o valor da pontuação para o critério escolhido em segundo lugar? Esse mesmo procedimento foi repetido com todos os critérios do modelo. Todas as taxas obtidas foram normalizadas dividindose as taxas brutas pela soma das taxas brutas fornecendo, portanto, resultados variando entre zero e 1.

O modelo mais comumente utilizado para a agregação das funções de valor local é o aditivo. Formalmente, pode ser assim definido pela seguinte formula:

$$
\begin{aligned}
& \text { ou } \\
& \mathrm{V}(a)=\sum_{\mathrm{i}}{ }^{\mathrm{n}} \mathrm{V}_{\mathrm{i}}(a) \cdot \mathrm{w}_{\mathrm{i}} \\
& \sum_{\mathrm{i}}{ }^{\mathrm{n}} \mathrm{w}_{\mathrm{i}}=1 \\
& 1>\mathrm{w}_{\mathrm{i}}>0 \forall \mathrm{i}
\end{aligned}
$$$$
\mathrm{V}(a)=\mathrm{v}_{1}(a) \cdot \mathrm{w}_{1}+\mathrm{v}_{2}(a) \cdot \mathrm{w}_{2}+. .+\mathrm{v}_{\mathrm{n}}(a) \cdot \mathrm{w}_{\mathrm{n}}
$$

Onde: $a=$ ação qualquer pertencente ao conjunto ' $\mathrm{A}$ ' de ações potenciais, $\mathrm{V}(a)=$ valor global de $a, \mathrm{v}_{\mathrm{i}}(a)=$ valor local (parcial) da ação $a$ segundo o critério $\mathrm{i}, \mathrm{w}_{\mathrm{i}}=$ taxas de harmonização do i-ésimo critério.

Tendo concluído os modelos, foram avaliados com os respectivos grupos, comparando com quatro sistemas alternativos de produção de batata orgânica.

Fase de elaboração de recomendações - Os resultados obtidos na fase de avaliação foram compilados e apresentados para validação pelos grupos de agricultores. Para isso, o modelo foi estruturado empregando-se uma planilha no software Excel para a geração de gráficos. Finalmente, foram realizadas duas reuniões com os demais agricultores de cada grupo com o objetivo de restituir os resultados do trabalho, avaliar os sistemas alternativos de produção de batata orgânica com apoio dos modelos e validar os sistemas de produção construídos com os agricultores em seus respectivos grupos.

\section{RESULTADOS E DISCUSSÃO}

O processo de construção do mapa cognitivo induziu ao aprendizado à reflexão e auxiliou na organização dos conhecimentos e percepções dos agri- 
Tabela 1. Comparação dos resultados dos modelos, com os critérios ordenados, seus respectivos pesos e a pontuação final (taxa normalizada) [comparison of model results, ordered with the criteria, their weights and the final score (standard rate)]. Pelotas, UFPel, 2012.

\begin{tabular}{|c|c|c|c|c|c|c|}
\hline \multirow{2}{*}{ Critérios (PVFs) } & \multicolumn{3}{|c|}{ Modelo 1} & \multicolumn{3}{|c|}{ Modelo 2} \\
\hline & Ordem & Peso bruto & Peso normalizado & Ordem & Peso bruto & Peso normalizado \\
\hline Tamanho da batata & $1^{\circ}$ & 100 & 0,120 & $4^{\circ}$ & 85 & 0,098 \\
\hline Risco de produção & $2^{\circ}$ & 95 & 0,114 & $1^{\mathrm{o}}$ & 100 & 0,116 \\
\hline Adubação orgânica & $3^{\circ}$ & 88 & 0,106 & $3^{\circ}$ & 90 & 0,104 \\
\hline Controle doenças e pragas & $3^{\circ}$ & 88 & 0,106 & $2^{\circ}$ & 95 & 0,110 \\
\hline Aparência da batata & $4^{\circ}$ & 85 & 0,102 & $3^{\circ}$ & 90 & 0,104 \\
\hline Gastos com insumos & $5^{\circ}$ & 75 & 0,090 & $5^{\circ}$ & 80 & 0,092 \\
\hline Escolha da área & $6^{\mathrm{o}}$ & 70 & 0,084 & $4^{\circ}$ & 85 & 0,098 \\
\hline Quant. de mão de obra & $7^{\circ}$ & 65 & 0,078 & $7^{\circ}$ & 50 & 0,058 \\
\hline Risco de comercialização & $7^{\circ}$ & 65 & 0,078 & $5^{\circ}$ & 80 & 0,092 \\
\hline Gastos com combustíveis & $8^{\circ}$ & 50 & 0,060 & $7^{\circ}$ & 50 & 0,058 \\
\hline Distribuição mão de obra & $8^{\circ}$ & 50 & 0,060 & $6^{\circ}$ & 60 & 0,069 \\
\hline
\end{tabular}

cultores sobre o problema da produção de batata orgânica. Aplicada a metodologia multicritério de apoio à decisão neste estudo, chegou-se aos seguintes resultados.

Na obtenção dos elementos primários de avaliação (EPAs), e posterior organização do processo de construção do mapa cognitivo, identificou-se o rótulo do problema por meio de indagação aos agricultores sobre os aspectos, que na visão deles, são importantes para a produção de batata orgânica. Para a obtenção dos EPAs, usou-se a técnica de brainstorming, com o propósito de extrair dos atores aqueles aspectos considerados, por eles, como relevantes à solução do problema apontado. $\mathrm{O}$ conjunto de EPAs foi agrupado em cinco grandes áreas: econômica (custos), técnica (produção), social (trabalho), padrão (qualidade) e riscos conforme verifica-se na Figura 1 do mapa cognitivo.

Sequencialmente procedeu-se à construção de conceitos a partir dos EPAs. Um conceito é uma idéia do EPA orientada à ação. Sendo expresso por dois pólos opostos entre si no significado psicológico. Normalmente, o primeiro polo simboliza a situação presente e o segundo designa um polo que, psicologicamente, para o decisor, é considerado contrário à situação atual. Os dois polos são separados por “...”" expressão que é lida "ao invés de". Portanto, o mapa deve ter uma perspectiva orientada à ação.

Finalmente, construiu-se a hierarquia de conceitos. A partir de um conceito o decisor pôde então ser questionado sobre os meios para obtêlo e sua importância. Para expandir o mapa em direção aos fins, questionou-se "por que esse conceito é importante?" e para expandi-lo em direção aos meios questionou-se "como obter esse conceito?".

A construção do mapa cognitivo possibilitou compreender as percepções sobre os aspectos relevantes da produção de batata no sistema orgânico, por meio das relações meio-fim entre os conceitos do mapa. No mapa do grupo da Rede de Referência (Figura 1), o conceito 11 "estar satisfeito com o sistema de produção de batata orgânica ... não estar satisfeito", foi identificado como sendo o único conceito "cabeça", ou seja, aquele em que todos os conceitos têm ligação, estando inserido os objetivos, fins, metas, valores e estratégias dos decisores para a obtenção dos resultados desejados. Neste mapa foram identificados 27 conceitos "rabos", que representam os meios, as ações, as alternativas que levam em direção aos objetivos mais estratégicos a serem alcançados.

Desta forma, o mapa do grupo 1 foi concluído contendo 77 conceitos e do grupo 2, com 80 conceitos, divididos em cinco áreas de interesse que também formam os clusters. Em cada uma das áreas ou clusters, foram identificadas as linhas de argumentação formadas por 11 ramos. Os ramos são constituídos por uma ou mais linhas de argumentação que demonstrem preocupações similares sobre o contexto decisório; trata-se, portanto, em uma análise de conteúdo que leva em conta as ideias expressas num conceito. Estes ramos foram enquadrados no contexto decisional de Keeney (1992) para a identificação dos Pontos de Vista Fundamentais, que são os critérios de avaliação do sistema de produção de batata orgânica.

Para ilustrar a construção do mapa cognitivo, na Figura 2 está reproduzido o clusters "produção" que é considerado o mais importante, por ser aquele que representa o eixo central da produção de batata. Nele foram identificados três ramos (R3, R4 e R5), que estão associados à escolha e preparo da área, assim como cuidados na compra da semente, o que requer atenção especial, pois a formação de uma boa lavoura depende fundamentalmente da qualidade e origem da semente; à adubação que é feita com o uso de adubos orgânicos e com adubação verde e ao controle das enfermidades enfatizando o controle de doenças como a requeima e a pinta-preta, que poderão causar enormes prejuízos à produção.

No cluster "custo", destacou-se aspectos relacionados aos insumos utilizados para a formação da lavoura como os fertilizantes, caldas fertiprotetoras para o controle de doenças e pragas, 


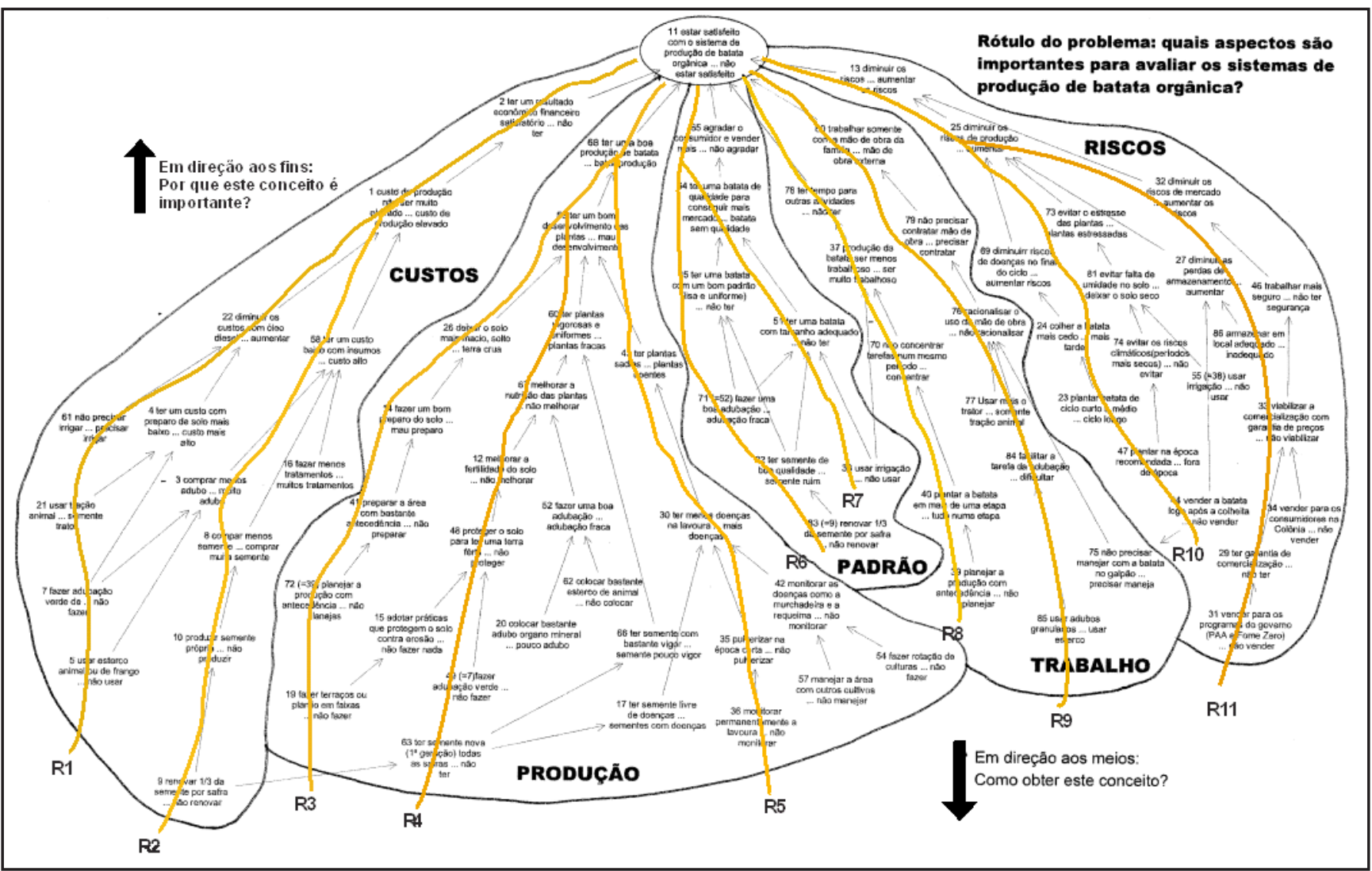

Figura 1. Mapa Cognitivo para avaliação de sistemas de produção de batata orgânica construído dos agricultores do Grupo da Rede de Referência. (cognitive map for evaluation of the production systems of organic potatoes built from farmers of the Group of Reference Network). Pelotas, UFPel, 2012.

sementes e combustíveis usados tanto para as operações de preparo de solo como para irrigação.

No cluster "padrão" e qualidade da batata, os agricultores deram ênfase aos aspectos da aparência e tamanho dos tubérculos. Estas características de qualidade foram destacadas pelos agricultores como sendo de muita importância para agradar o consumidor final.

No cluster "trabalho", foi avaliado o uso e a distribuição da mão de obra para o cultivo da batata, visando planejar a produção de maneira que possam executar todas as tarefas com a mão de obra disponível na família. O plantio em frações de áreas escalonadas é importante também para que sejam cuidadas adequadamente e que não sobrecarreguem a família e nem tampouco deixem de fazer outras atividades em detrimento da concentração de tarefas no cultivo da batata.

No cluster "riscos" da produção, foram destacadas três áreas bem definidas. Riscos ambientais relacionados ao clima, riscos de produção vinculados aos aspectos de deficiência hídrica, doenças e pragas, perdas no armazenamento e, por último, riscos associados aos processos de comercialização.

Os pontos de vista fundamentais e elementares, identificados após o enquadramento dos ramos do mapa cognitivo, foram agrupados de acordo com os clusters identificados. Examinando as linhas de argumentação que formam os ramos do mapa, observou-se que em cada um deles havia mais de um aspecto a ser considerado. Deste modo, foi efetuada uma subdivisão dos PVFs em PVEs com o objetivo de melhorar a descrição dos impactos das ações a serem avaliadas no processo de produção de batata orgânica (Figura 3). Também, foram inseridos nesta figura as taxas de compensação dos subcritérios e critérios obtidos por meio do método Swing Weights (Gomes, 2001) e observam-se as preferências em torno dos critérios (PVFs), gerando um ranking do mais importante (peso maior) ao menos importante (peso menor), segundo a avaliação dos agricultores.

Comparando-se os dois grupos, evidenciam-se algumas diferenças tanto na descrição dos critérios, como na ordenação das preferências. Em relação ao cluster "custos", a maior diferença recaiu sobre o critério custo da semente. Para o grupo 1, a elevação do custo na aquisição de sementes para a renovação de $1 / 3$ ou mais por safra, representa uma rejeição maior em relação ao grupo 2 . Por sua vez, em relação aos insumos orgânicos, estabeleceu-se uma diferença ocasionada pelo uso de produtos comerciais em detrimento do uso de insumos produzidos na propriedade. Neste caso, o grupo 1 teve gastos mais elevados que o grupo 2 .

No cluster "produção", que envolveu a análise do tipo e preparo da área, do uso de fertilizantes orgânicos e do controle de pragas e doenças, a maior diferença se verificou no subcritério origem da semente, que para o grupo 2 , se a mesma não for certificada, representa 


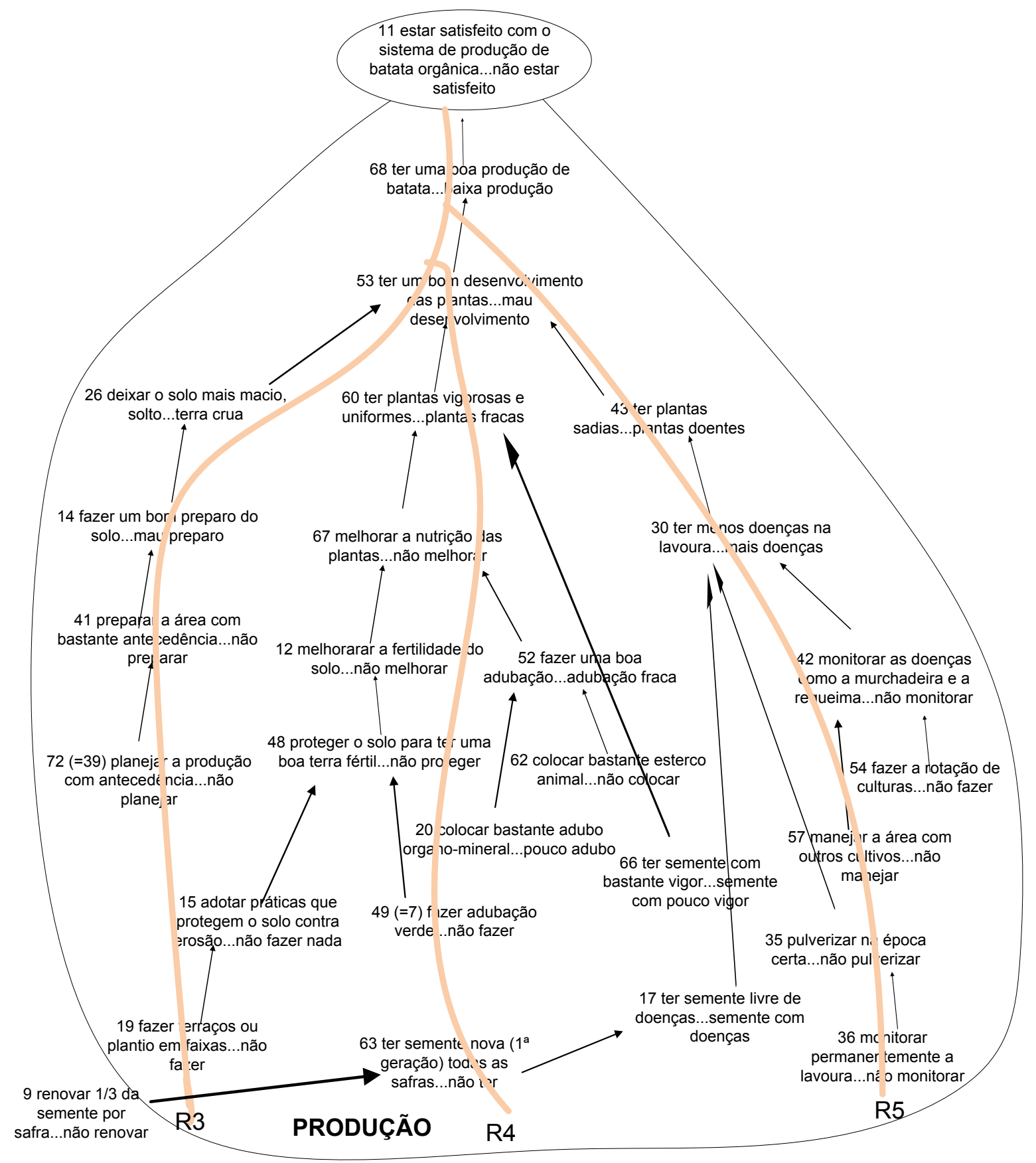

Figura 2. Cluster "produção" do mapa cognitivo para avaliação de sistemas de produção de batata orgânica construído dos agricultores do Grupo da Rede de Referência ("production" cluster of the cognitive map for evaluation of the production systems of organic potatoes built from farmers of the Group of Reference Network). Pelotas, UFPel, 2012.

uma rejeição elevada. Para o grupo 1, este tipo de semente é aceitável. Neste cluster, o que ficou evidenciado para os dois grupos, foram as escolhas no nível "bom", ou seja, para o grupo 1, as melhores terras para o cultivo da batata, são de pousio, e para o grupo 2, as de campo nativo. Para os dois grupos, para um bom preparo do solo, são necessárias quatro operações, usar gramíneas e leguminosas para a adubação verde, e as cultivares devem apresentar boa resistência à requeima e realizar o controle de doenças e pragas com cinco tratamentos. Quanto à adubação, usou-se como parâmetro a proporção de organo-mineral e esterco de aves em relação à semente, da seguinte maneira: grupo $1>0,6 \mathrm{~kg}$ de adubo $+1,3 \mathrm{~kg}$ de esterco/1,0 $\mathrm{kg}$ de semente; grupo $2>0,75 \mathrm{~kg}$ de adubo + $1,5 \mathrm{~kg}$ de esterco/1,0 kg de semente.

No cluster "padrão", que avaliou a qualidade da batata pelos critérios aparência e tamanho, para o grupo 1, 


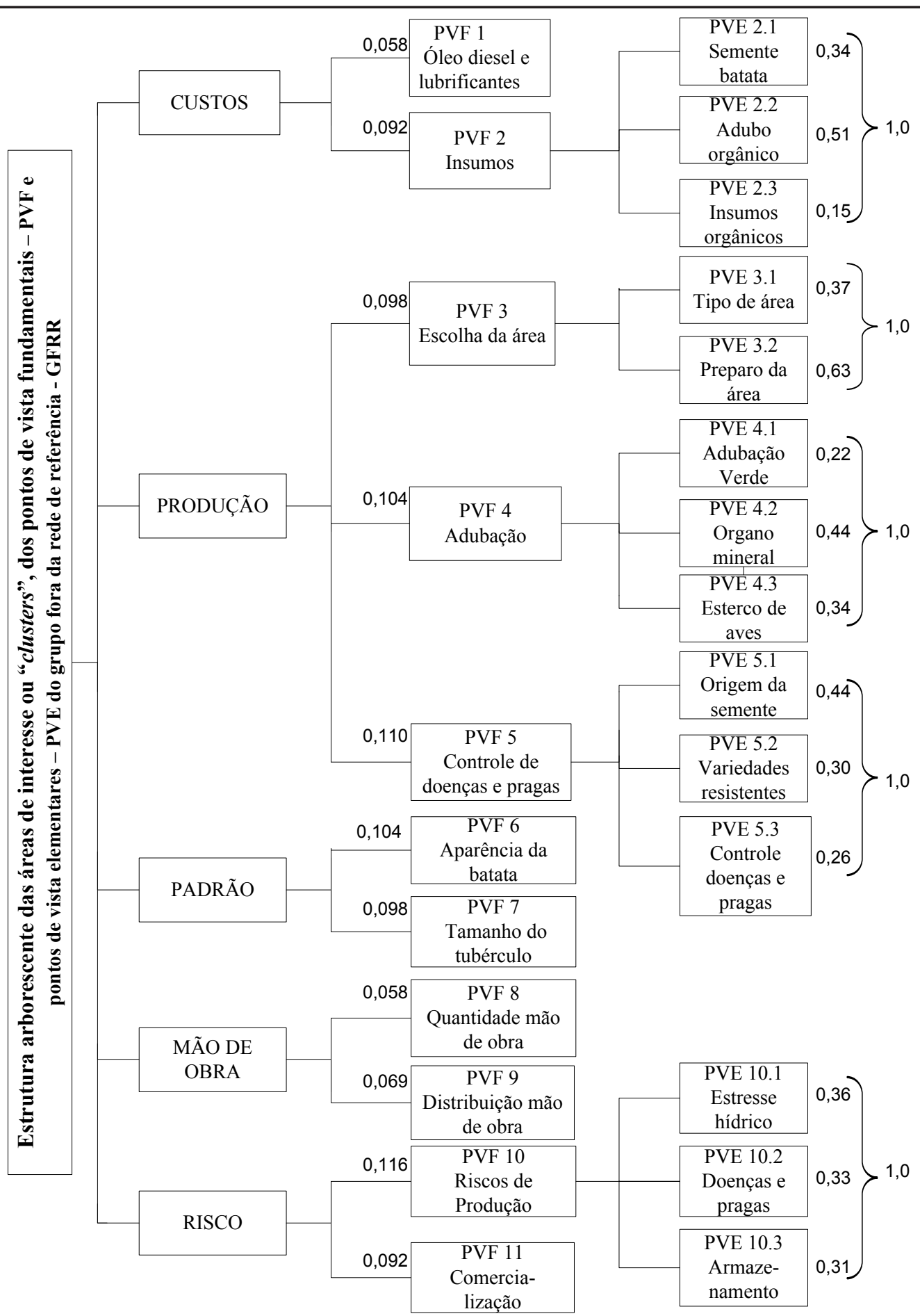

Figura 3. Estrutura arborescente formada pelos Pontos de Vista Fundamentais (PVFs) e Pontos de Vista Elementares (PVEs), taxas de compensação normalizadas por PVE e PVF, para avaliação de sistemas de produção de batata orgânica do Grupo Fora da Rede de Referência (GFRR) [tree structure formed by the Fundamental Points of View (PVFS) and Points of View Elementary (PVEs), compensation rates standardized by PVE and PVF for evaluation of the production systems of organic potatoes of the Group Outside the Reference Network (GFRR)]. Pelotas, UFPel, 2012.

a obtenção de até $10 \%$ de batatas descartáveis representa o nível "neutro" e acima deste percentual, a rejeição elevase. Para o Grupo 2, situa-se dentro da região de expectativa. Para este grupo, colher até $15 \%$ de batata descartável, representa a situação "neutra" e acima deste percentual, a situação não é desejável. Em relação ao critério tamanho dos tubérculos, para o grupo 1, obter produção de no máximo $25 \%$ de batata com tubérculos não aceitos comercial- mente representa o índice de rejeição ("neutro"), ao passo que para o grupo 2 , o percentual aceitável é até $50 \%$.

No cluster "trabalho", foi avaliado o uso da mão de obra na quantidade e distribuição. Verificou-se que as maiores 


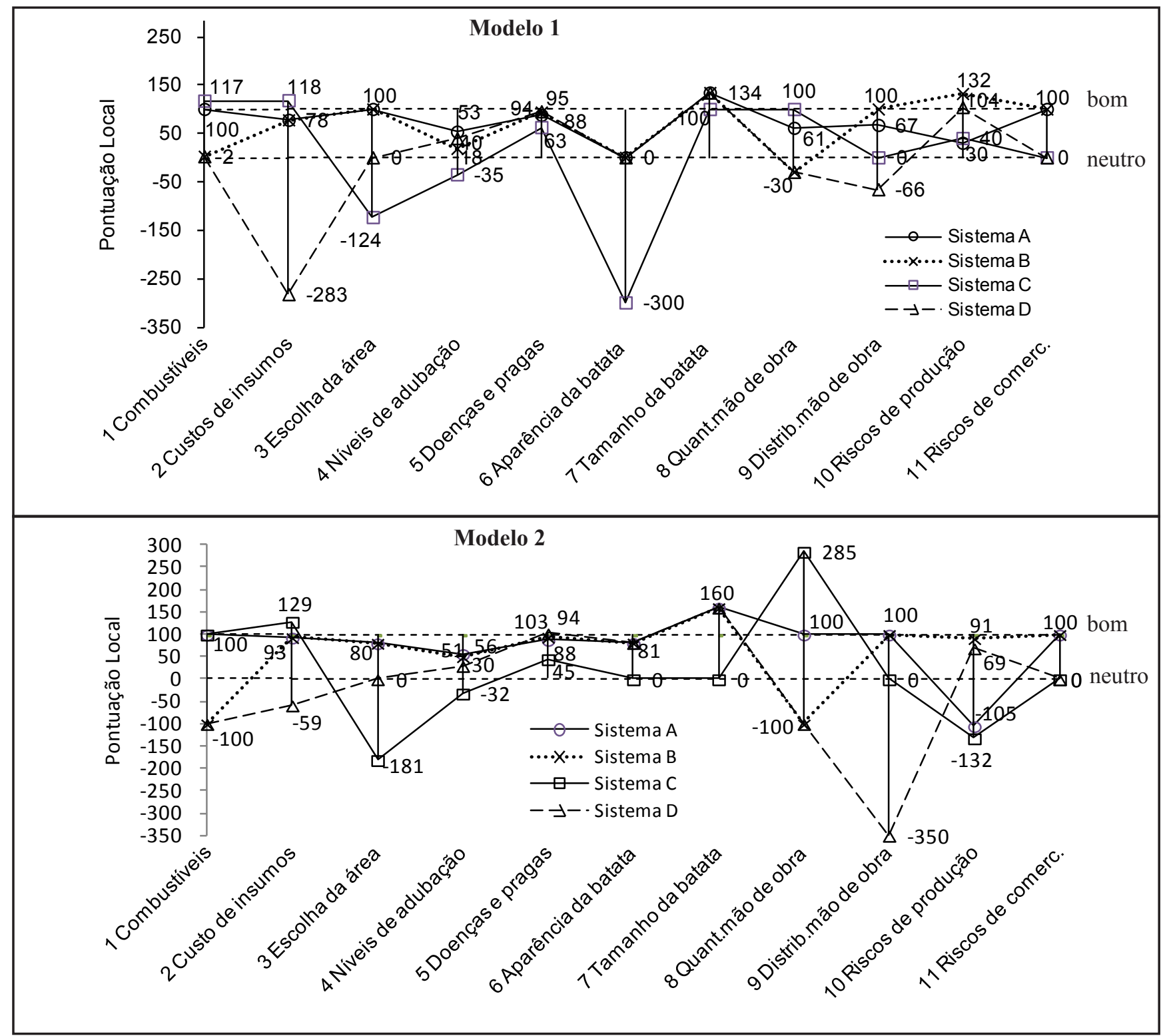

Figura 4. Níveis de impacto máximos e mínimos dos pontos de vistas fundamentais (critérios) dos quatro sistemas alternativos de produção de batata "A, B, C, D" avaliados com os modelos 1 e modelo 2 [levels of maximum and minimum impact of fundamental points of view (criteria) of the four alternative systems of potato production “A, B, C, D" evaluated with models 1 and model 2]. Pelotas, UFPel, 2012.

diferenças entre os dois grupos residem nas quantidades de dias/homem trabalhados para a produção de um hectare de batata, muito em função do uso da irrigação utilizada pelo Grupo 2. A operação de irrigação exige muita mão de obra na distribuição dos equipamentos e cuidados durante a operação. De qualquer forma, este critério é o que representa a menor restrição ao sistema de produção de batata, seja na quantidade e no esforço físico, ou seja, não representa trabalho penoso em nenhuma das fases de cultivo para os dois grupos.

No cluster "riscos" é descrito o grau de riscos em que a produção de batata está exposta em decorrência de elementos climáticos, doenças e pragas e de mercado. Neste critério, verificouse algumas diferenças bem acentuadas nos níveis de impactos entre os grupos. A maior reside no subcritério "estresse hídrico", que para o grupo 1, fazer uma irrigação na safra já é considerado "Bom", enquanto para o Grupo 2, o nível "Bom" é realizar acima de três irrigações. Outra diferença verificada neste critério foi em relação ao subcritério controle de doenças. Para o grupo 1 , o uso de sementes não certificadas representa um risco elevado, enquanto para o grupo 2, desde que monitorada a lavoura, representa um risco aceitável. A terceira diferença neste cluster reside no critério comercialização. Enquanto para o grupo 1, ter apenas uma opção de mercado, representa um índice de rejeição baixo e para o Grupo 2, é considerada elevada. Ambos os grupos consideram que três opções são o ideal.

A avaliação final em cada um dos grupos apresentou pequenas, mas importantes alterações tanto na ordem quanto na pontuação (Tabela 1). No modelo 1 , a ordem preferencial es- 
tabelecida determinou em $1^{\circ}$ lugar, o critério "Tamanho da batata", enquanto no modelo 2, foi o critério "Riscos de produção". Estes resultados demonstram o equilíbrio existente entre os dois grupos ao ordenar e estabelecer um grau de importância em cada um dos critérios na avaliação do sistema de produção de batata orgânica de acordo com as percepções e preferências dos agricultores que a metodologia MCDA pode captar.

Os dois modelos foram submetidos a uma avaliação com seus respectivos grupos, confrontando-os com quatro sistemas de produção de batata orgânica. Estes sistemas foram identificados como sistemas “A, B, C, D”. O sistema A, preconiza o uso dos parâmetros tecnológicos adotados pelo grupo 1 . $\mathrm{O}$ sistema $\mathrm{B}$ incorpora as práticas e manejo realizados pelo grupo 2 . O sistema $\mathrm{C}$ é um alternativo utilizado por agricultores que utilizam um nível tecnológico mais baixo em relação aos sistemas $\mathrm{A}$ e B. O sistema D é o que representa e se assemelha ao utilizado e recomendado pela pesquisa da Embrapa Clima Temperado, com um aporte tecnológico mais elevado, principalmente no que diz respeito ao uso de fertilizantes e de caldas fertiprotetoras.

$\mathrm{Na}$ Figura 4, pode-se verificar que os perfis de impacto nos níveis máximos e mínimos dos critérios em cada sistema de produção nos dois modelos, obtidos por intermédio da fórmula de agregação aditiva descrita na metodologia apresentam proximidades nos sistemas A e B, onde a maioria dos pontos encontra-se dentro da região de expectativa. Por outro lado, nos sistemas C e D os níveis de impacto apresentam muitos pontos negativos, tanto no modelo 1 quanto no modelo 2. Os impactos que se posicionaram nas maiores extremidades negativas foram nos sistemas $\mathrm{C}$ e $\mathrm{D}$, sendo que no modelo 1 , os custos com insumos representaram a maior pontuação para o sistema D (-283) e a aparência da batata para o sistema C (-300). Por sua vez no modelo 2, a distribuição da mão de obra (-350) foi o critério que mais impactou negativamente no sistema $\mathrm{D}$, e a escolha da área (-181) e os riscos de produção (-132) no sistema C. Por outro lado, na ponta de cima, no critério tamanho da batata teve um empate triplo com três sistemas obtendo a mesma pontuação (134), demonstrando um equilíbrio destes sistemas neste critério.

Desta análise pode ser concluído que os sistemas C e D tiveram uma rejeição maior nos dois grupos, influenciado por aspectos bem opostos. O sistema $\mathrm{C}$ apresenta uma situação atrativa quanto aos baixos custos de produção e um menor uso de mão de obra; no entanto, apresenta índices negativos em relação à escolha da área, baixa fertilização, baixa qualidade de batata e consequentemente altos riscos de produção. Em relação ao sistema D, apresenta critérios atrativos, como a fertilização, o controle de doenças e pragas, e a colheita de uma batata de qualidade. No entanto, para se obter estes índices de produção, os custos com insumos e mão de obra são elevados, o que decorre em rejeição pelos agricultores.

Os dois modelos construídos com os agricultores, quando avaliados com outros quatro sistemas hipotéticos de produção de batata orgânica, identificados como (A, B, C, D) tiveram desempenhos muito próximos na avaliação global. No modelo 1, as pontuações globais foram de 72,6, 70,2, 4,1 e 10,9 e no modelo 2, $71,7,71,3,2,7$ e 5,0 respectivamente. $\mathrm{Na}$ avaliação de cada um dos grupos, os agricultores concordaram com os resultados obtidos nos modelos, comprovando sua validade e robustez frente aos modelos construídos. Este resultado demonstra um grau de conhecimento apurado e sintonizado dos agricultores ao avaliar os modelos de produção de batata orgânica dos dois grupos.

Os sistemas C e D distanciaram-se na avaliação global dos sistemas A e $B$ em função do aporte de tecnologias usadas. Na avaliação realizada com os agricultores dos dois grupos, eles afirmaram que o uso de muita tecnologia nem sempre significa a obtenção de altas produções, porque, faz parte deste processo de produção um conjunto de elementos e não apenas uma ação isolada. Por isso, no grupo 1, a preferência recaiu sobre o sistema A, sendo escolhido em primeiro lugar por $57 \%$ dos agricultores, seguido do sistema $B$, em segundo lugar por $28 \%$, enquanto no grupo 2 , o sistema $\mathrm{B}$, foi escolhido em primeiro lugar por $50 \%$ dos agricultores e o sistema $\mathrm{A}$, em segundo lugar por $41,7 \%$. Baseado nesta avaliação pode-se concluir que os agricultores validaram os dois modelos construídos com os agricultores.

Este estudo com os resultados completos pode ser vistos em Reichert (2012) e sugere que há aspectos importantes a serem considerados e analisados no processo de produção de batata orgânica da região Sul do RS. Independentemente do mercado consumidor, a batata deve apresentar boa qualidade estética em relação ao tamanho, aparência, isenta de manchas, danos causados por insetos ou mecânicos e defeitos fisiológicos. Para conseguir-se uma produção de qualidade, faz-se necessário prevenir contra alguns fatores de riscos como estiagem prolongada, escolha correta da semente, controle de pragas e doenças, entre outros.

Independentemente do sistema de produção da batata, um aspecto importante na cadeia é a comercialização. Apesar do conhecimento e domínio na produção de batata, os agricultores carecem ainda de tecnologias alternativas viáveis e compatíveis com a infraestrutura da unidade produtiva, em relação ao manejo da fertilização, manejo de doenças e pragas, manejo de irrigação, bem como aspectos ligados à comercialização. Neste sentido, é recomendável que Instituições de Pesquisa, extensão e de apoio administrativo e organizacional, desenvolvam ações na direção dos agricultores, para tornar a bataticultura mais sustentável dos pontos de vista social, ambiental e econômico.

\section{REFERÊNCIAS}

ALTIERI M. 1998. Agroecologia: a dinâmica produtiva da agricultura sustentável. Porto Alegre: Editora da UFRGS. 110p.

ANDERSON NLM. 2010. Seleção de Tratores Agrícolas Adequados à Agricultura Familiar. Pelotas: UFPel. 111p. (Dissertação mestrado).

ARCE FA. 2002. El cultivo de la patata. 2. ed. Madrid: Ediciones Mundi-Prensa. 495p.

BEINAT E. 1995. Multiattribute value functions for environmental management. Amsterdam: Timbergen Institute Research Series.

BOUYSSOU D. 1990. Building criteria: a prerequisite for MCDA. In: BANA \& COSTA CA (eds). Readings in multiple criteria decision aid. Berlin: Springer-Verlag.

COARACY V. 1957. A Colônia de São Lourenço e seu Fundador Jacob Rheingantz. São Paulo: Oficinas Gráficas Saraiva SA. 139p. 
COSTA JS. 1984. Origens históricas do município de São Lourenço do Sul. In: São Lourenço do Sul - Cem Anos 1884 - 1984. São Lourenço do Sul. p. 39-77.

EDEN C. 1989. Using cognitive mapping for strategic options development and analysis (SODA). In: ROSENHEAD J. (ed) Rational analysis for a problematic world: problem structuring methods for complexity, uncertainty and conflict. Chicester: J Wiley \& Sons. p. 21-42.

ENSSLIN L; MONTIBELLER NETO G; NORONHA S. 2001. Apoio à decisão metodologia para estruturação de problemas $e$ avaliação multicritério de alternativas. Florianópolis: Insular. 296p.

FNPCONSULTORIA\&AGROINFORMATIVOS. 2011. São Paulo: AgraFNT, Agrianual 2011. Anuário da agricultura brasileira. São Paulo, $482 \mathrm{p}$.

GOMES MC. 2001. Apoio à decisão em empresas familiares em processo de evolução: um modelo multicritérios em um estudo de caso na indústria de conservas de Pelotas-RS. Florianopolis: UFSC. 417p. (Tese doutorado).

IBGE/SIDRA. Sistema IBGE de Recuperação Automática: Banco de Dados Agregados. Disponível em: <http://www.sidra.ibge.gov. br>. Acesso em: 12 maio 2012

KEENEY RL. 1992. Value-focused thinking: a path to creative decision making. Cambridge: Harvard University Press. 416p.
LEFF H. 2002. Agroecologia e saber ambiental. Agroecologia e Desenvolvimento Rural Sustentável 3: 36-51.

LIMA MIF. 2006. Paisagem, terroir e sistemas agrários: um estudo em São Lourenço do Sul. Porto Alegre: UFRGS. 151p. (Dissertação mestrado).

MADAIL JCM; PEREIRA AS; SIMA LF. 2005. Agronegócio da batata no sul do RS. Pelotas: Embrapa Clima Temperado. 30p. (Boletim de Pesquisa e Desenvolvimento, 18).

MARTINEZ EA. Caracterização do sistema de produção de batata em transição agroecológica de agricultores familiares em São Lourenço do Sul (RS). 2009. 109p. Pelotas: UFPel. (Dissertação mestrado).

MEDEIROS CAB; REICHERT LJ; GOMES JCC; HEBERLÊ ALO. 2005. Tecnologias para os Sistemas de Produção e Desenvolvimento Sustentável da Agricultura Familiar - Projeto RS Rural. Pelotas: Embrapa Clima Temperado. $89 \mathrm{p}$.

MONTIBELLER NETO G. 1996. Mapas cognitivos: uma ferramenta de apoio à estruturação de problemas. Florianópolis: UFSC. 205p. (Dissertação mestrado).

NAZARENO NRX; JACCOUD FILHO DS. 2003. Doenças fúngicas. In: PEREIRA AS; DANIELS J (eds). O cultivo da batata na Região Sul do Brasil. Brasília: Embrapa Informação Tecnológica. p.239-276.
NAZARENO NRX; PEREIRA. AS. 2009. Cultivares de batata adaptadas ao sistema orgânico de produção. In: NAZARENO NRX (ed). Produção orgânica de batata potencialidades e desafios. Londrina: IAPAR. p. 109-119.

PEREIRA AS; DANIELS J. 2003. O cultivo da batata na região sul do Brasil. Embrapa Clima Temperado. - Brasília, DF: Embrapa Informações Tecnológicas.

REICHERT LJ. 2012. Avaliação de sistemas de produção de batata orgânica em propriedades familiares: uma aplicação da metologioa multicritério de apoio à decisão (MCDA). Pelotas: UFPel. 346p. (Tese doutorado).

ROSSI F. 2009. Cultivares para o Sistema Orgânico de Produção de Batata. Piracicaba: ESALQ-USP. 89p. (Tese doutorado).

ROY B. 1993. Decision.science or decision-aid science? European Journal of Operational Research 66: 184-203.

ROY B; VANDERPOOTEN D. 1996. The European school of MCDA: emergences, basic features and current works. Journal of Multicriteria Decision Analysis 5: 23-38.

XAVIER JHV. 2010. Avaliação de sistemas de cultivo de milho grão sequeiro no contexto da agricultura familiar: uma aplicação da metodologia multicritério de apoio à decisão (MCDA). Pelotas: UFPel. 318p. Pelotas. (Tese doutorado). 